\title{
Fiche technique de l'impact de l'amendement du substrat en poudre de coquille d'ouf de poule sur la reproduction de Archachatina marginata (Swainson, 1821)
}

\author{
Aman Jean Baptiste \\ Memel Jean Didier
}

Université Nangui Abrogoua, UFR-Sciences de la Nature, Laboratoire de Biologie et Cytologie Animale, Pôle de recherche Productions Animales,

Côte d'Ivoire

\section{Kouassi Kouadio Daniel}

Université Jean Lorougnon Guédé, Département de biologie et de physiologie Animale, UFR Environnement, Daloa, Côte d'Ivoire

\section{Otchoumou Atcho}

Université Nangui Abrogoua, UFR-Sciences de la Nature, Laboratoire de Biologie et Cytologie Animale, Pôle de recherche Productions Animales, Côte d'Ivoire

Doi: 10.19044/esj.2018.v14n33p239 URL:http://dx.doi.org/10.19044/esj.2018.v14n33p239

\begin{abstract}
This study consists in looking for an improvement of Archachatina margina reproductions performances by amendment of the breeding substrates. As a result, five types of substrate were prepared by soil amendment at different rates $(0 \%, 10 \%, 20 \%, 30 \%$ et $40 \%)$ with chicken egg shell powder. On these substrates, snails were raised from spat stage until they were laid. Reproductive parameters such as: age of first laying, the number of annual laying per animal, the number of eggs per laying on the different substrates were recorded. Overall, snail laying performances improved with increasing rate of substrate amendment. However, beyond a rate of $20 \%$, the weight of eggs laid began to decline. The chicken egg shell powder is suitable for the amendment of the breeding substrate of snails Archachatina marginata in view of an improvement in their reproductive performance. The rate of amendment recommended to this effect is $20 \%$.
\end{abstract}

Keywords: Archachatina marginata, amendment, substrate, reproductive performance 


\section{Resume}

Cette étude à consister à rechercher une amélioration des performances de reproduction de Archachatina marginata par amendement des substrats d'élevage. De ce fait, cinq types de substrat ont été confectionnés par amendement du terreau à différents taux $(0 \%, 10 \%, 20 \%$, $30 \%, 40 \%$ ) en poudre de coquille d'œufs de poule. Sur ces substrats, des escargots ont été élevés du stade naissain jusqu'à leur ponte. Les paramètres de reproduction tels que : l'âge de première ponte, le nombre de ponte annuel par animal et le nombre d'œufs par ponte sur les différents substrats ont été enregistrés. Dans l'ensemble, les performances de ponte des escargots se sont améliorées avec l'augmentation du taux d'amendement de leur substrat. Cependant, au-delà d'un taux de $20 \%$, le poids des œufs pondu a commencé à baisser. La poudre de coquille d'œuf de poule convient à l'amendement du substrat d'élevage des escargots Archachatina marginata en vu d'une amélioration de leur performance de reproduction. Le taux d'amendement conseillé à cet effet est de $20 \%$.

Mots clés: Archachatina marginata, amendement, substrat, performances de reproduction

\section{Introduction}

L'élevage des escargots géants africains, connaît de plus en plus un essor en Afrique subsaharienne. Cependant, cet essor reste tributaire de véritables techniques d'élevage susceptibles de non seulement réduire le cycle de croissance et de reproduction mais aussi, d'améliorer les performances de ponte des escargots. Ainsi, la maîtrise de l'achatiniculture nécessite, une modernisation des techniques d'élevage (Rousselet, 1982; Chevalier, 1985; Bonnet et al., 1990). C'est ce qui justifie les nombreux travaux réalisés ces dernières années pour déterminer des régimes alimentaires et des substrats convenables pour l'élevage de ces mollusques (N'da et al., 2004; Kouassi et al., 2007; Diomandé et al., 2008; Otchoumou et al., 2011 ; Aman et al., 2011 ; Bouye et al., 2015 et Sika et al., 2015).

Des travaux ont montré que le calcium est un élément important dans la répartition des escargots en milieu naturel (Johannessen, 2001 ; Tattersfield et al., 2001 ; Hotopp, 2002). Les escargots utilisent ce minéral pour la confection de leur coquille et la calcification de leurs œufs (Bonnet et al., 1990). C'est à juste titre que les régions où vivent ces mollusques, possèdent un sol calcaire. 
Vu l'important rôle que joue le calcium du sol chez l'escargot, il conviendrait de rechercher une source calcique qui contiendrait du calcium bio-disponible pour l'amendement du substrat de ces animaux en milieu d'élevage. Cette étude a donc pour but de rechercher une amélioration des performances de reproduction de Archachatina marginata par amendement du sol en calcium avec de la poudre de coquille d'œufs de poules.

\section{Materiel et Methodes}

\section{Matériel biologique}

\section{Reproducteurs}

Les reproducteurs considérés dans cette étude sont des jeunes reproducteurs de Archachatina marginata élevés depuis le stade naissains jusqu'à leur maturité sexuelle (Figure 1).

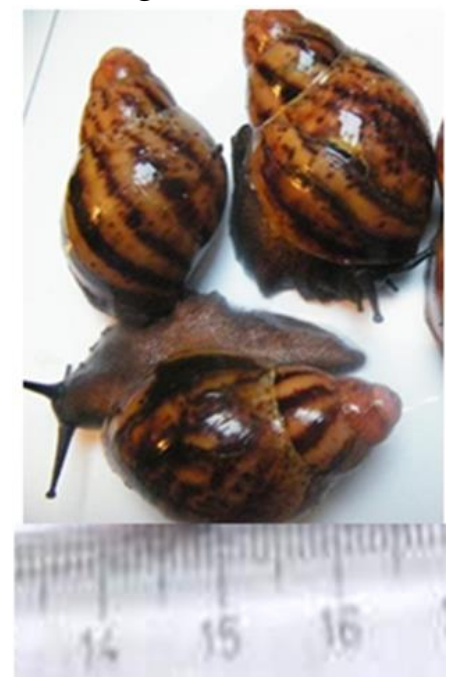

Figure1 : Reproducteurs Archachatina marginata

\section{Aliment}

Les animaux ont été nourris exclusivement à l'aliment concentré sous la forme farine (Figure 2) formulé sur la base des travaux de Conan et al. (1989).

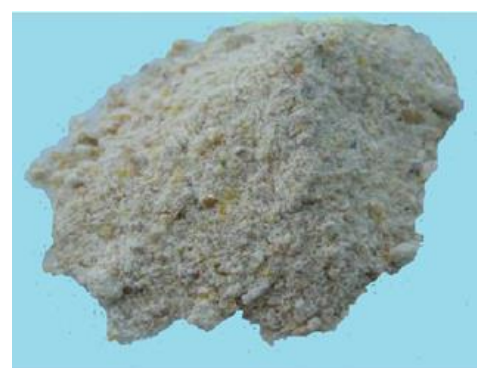

Figure 2: Aliment concentré des escargots 


\section{Matériel d'élevage}

\section{Bacs d'élevage}

Des bacs en plastique de forme parallélépipédique de dimensions 0,8 x $0,75 \times 0,8 \mathrm{~m}$, soit une surface de base de $0,6 \mathrm{~m}^{2}$ pour un volume de $0,48 \mathrm{~m}^{3}$, ont servi d'enceinte pour l'élevage des escargots (Figure 3). Ces bacs sont munis de couvercles de type moustiquaire constituant le dispositif anti-fuite et permettant la circulation d'air. Ils ont été installés dans un bâtiment à toiture recouverte de tôles et muni d'un lanterneau asymétrique.

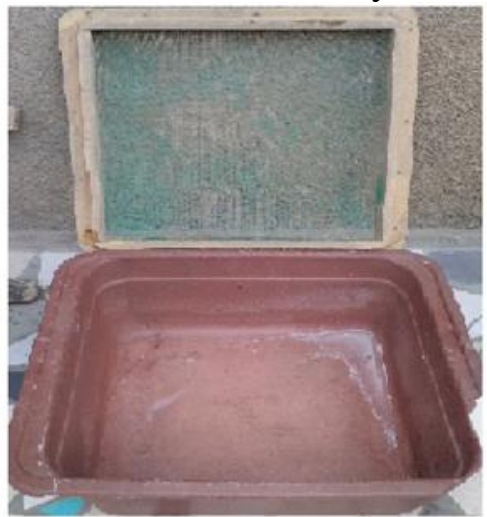

Figure 3 : Bac d'élevage

\section{Bacs d'incubation}

L'incubation des œufs a été effectuée dans des bacs en plexiglas à base carrée et à faces trapézoïdales. Ils ont une hauteur de $0,14 \mathrm{~m}$ avec une petite base de côté $0,17 \mathrm{~m}$ et une grande base de côté $0,21 \mathrm{~m}$, soit une surface de base de $28910^{-4} \mathrm{~m}^{2}$ (Figure 4). Ces bacs perforés à la base sont munis de couvercles aussi perforés qui adhèrent à leurs pourtours. Les perforations permettent à l'air de circuler librement dans ces bacs. Aussi, ces perforations permettent d'évacuer l'excès d'eau d'arrosage.

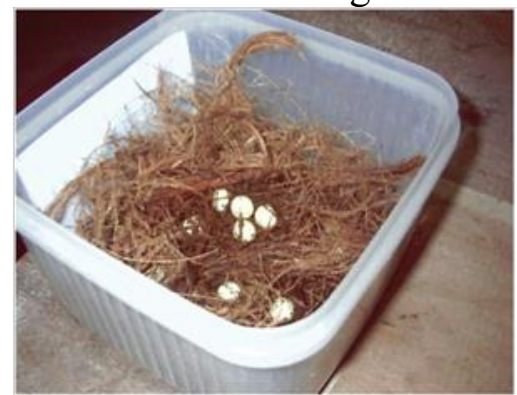

Figure 4 : Bac garni de substrat d'incubation

\section{Substrat d'incubation}

Le substrat d'incubation des œufs est confectionné avec des bourres de noix de coco séchés. Le choix de ce substrat est guidé par les travaux de Aman 
(2004) qui ont révélé que celui-ci permet d'optimiser le taux d'éclosion des œufs des escargots du genre Archachatina (Figure 4).

\section{Source calcique}

Les coquilles d'œufs de poules ont été utilisées comme source calcique pour l'amendement des substrats d'élevage.

\section{Sol}

Du terreau prélevé à la surface du sol de la forêt de l'université Abobo Adjamé (Actuelle Université Nangui Abrogoua) a été utilisé comme élément de base pour la confection du substrat d'élevage.

\section{Matériel technique}

\section{Balance}

Une balance électronique de marque Sartorius de précision $0,1 \mathrm{~g}$ a été utilisée pour peser les œufs et les éléments constitutifs (sources calciques et terreau) des substrats d'élevage.

\section{Pied à coulisse}

Un pied à coulisse mécanique de précision $0,1 \mathrm{~mm}$ a servi pour la mensuration des diamètres des œufs.

\section{Mangeoire et abreuvoir}

Des fermetures de grandes boites de lait en plastique à base extérieur tapissée d'une couche de ciment ont été utilisées comme mangeoires et abreuvoirs (Figure 4). La couche de ciment permet d'augmenter la masse du plastique afin d'éviter que les animaux renverse le contenu.

\section{Méthodes}

\section{Traitement du sol}

Du terreau a été prélevé en surface du sol à une profondeur d'au plus $10 \mathrm{~cm}$. Après prélèvement il a été tamisé pour être débarrassé des morceaux de bois et des pierres. Après le tamisage celui-ci a été légèrement arrosé pour humidification ensuite chauffé dans une bassine en aluminium sur du biocharbon pendant 20 minutes. Le chauffage permet de débarrasser le terreau des œufs d'insectes (Alluaudihella flavicornis, fongus Fusarium) pouvant s'attaquer aux escargots.

\section{Traitement de la source calcique}

Des coquilles d'œufs de poule ont été collectées dans des kiosques à café. Elles ont ensuite été trempées dans de l'eau bouillante pour désinfection. Ces coquilles ont par la suite été concassées et chauffées à l'étuve à $70^{\circ} \mathrm{C}$ 
pendant 8 heures. Après chauffage elles ont été transformées en poudre au mortier.

\section{Amendement du terreau}

Le terreau a été amendé avec la poudre de coquilles d'œufs obtenue à différent taux $(5 \%, 10 \%, 20 \%, 30 \%$ et $40 \%)$ comme l'indique la figure 5 . Un substrat non amendé constitué à $100 \%$ de terreau a été considéré comme témoin. Ainsi, cinq types de substrats $\left(\mathrm{S}_{\mathrm{T}}, \mathrm{S}_{\mathrm{oe} 5}, \mathrm{~S}_{\mathrm{oe} 10}, \mathrm{~S}_{\mathrm{oe} 20}, \mathrm{~S}_{\mathrm{oe} 30}\right.$ et $\mathrm{S}_{\mathrm{oe} 40)}$ ont été confectionnés en raison de 3 répétitions par traitement.

\section{Disposition du substrat}

Les différents substrats confectionnés sont disposés, chacun au planché des bacs jusqu'à une hauteur de $20 \mathrm{~cm}$, permettant ainsi aux animaux d'enfouir aisément leurs œufs. Ces substrats sont ensuite recouverts d'une couche d'épaisseur $5 \mathrm{~cm}$ de feuilles sèches de cacaoyers. Ces feuilles ont au préalable été désinfectées par trempage dans de l'eau bouillante.

\section{Disposition des escargots}

Les escargots ont été répartis au stade naissains (à 2 semaines d'âge) à une densité de 25 individus par $\mathrm{m}^{2}$ dans les bacs d'élevage sur les différents substrats.

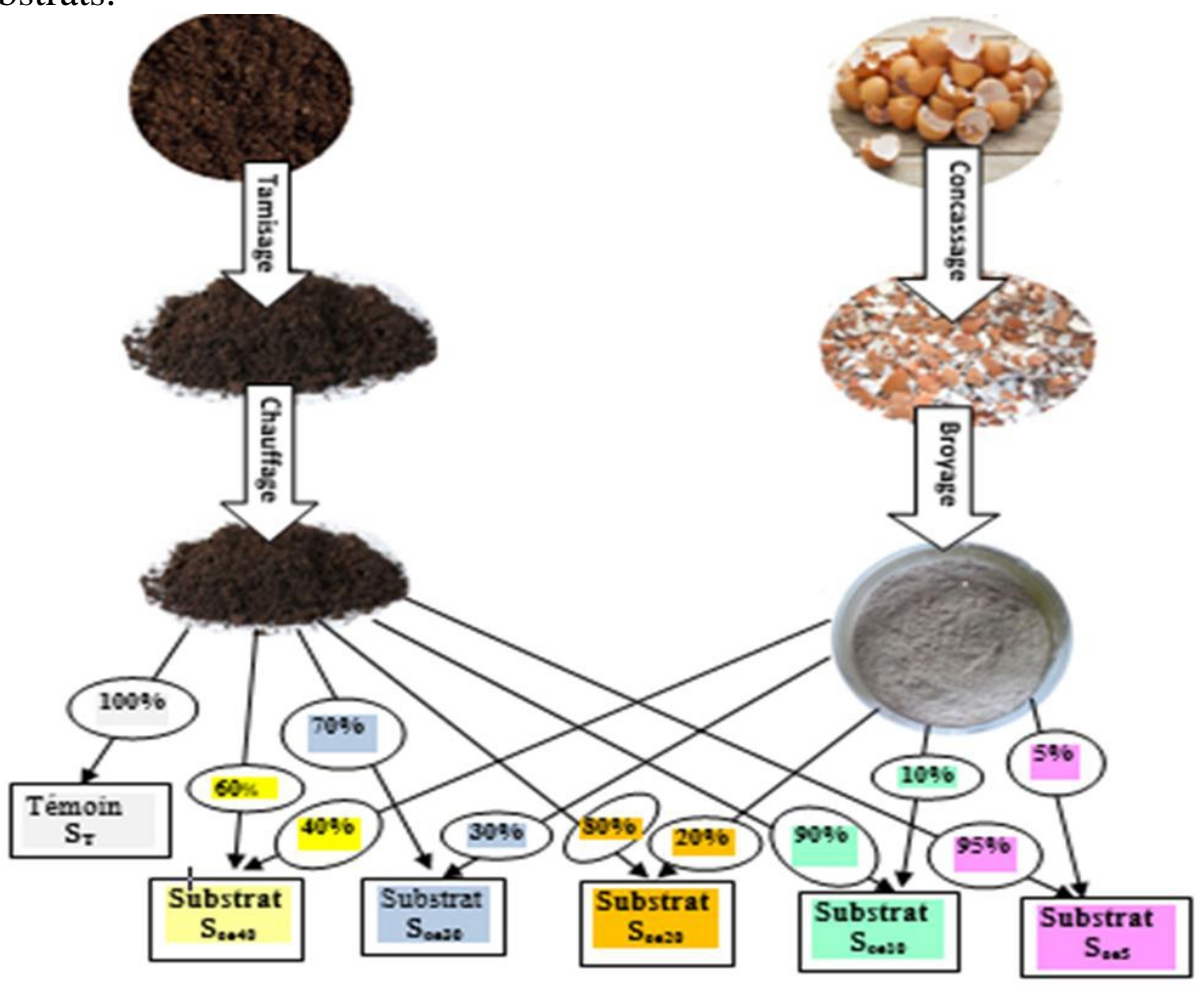

Figure 5 : Méthode de confection des substrats d'élevage 


\section{Alimentation des escargots}

Les escargots ont été nourris à volonté tous les deux jours. Au terme des deux jours, les mangeoires étaient correctement lavées et séchés avant d'être réutilisées. Aussi, de l'eau potable leur a été distribuée dans les abreuvoirs quotidiennement. Avant de remplir les abreuvoirs, ceux-ci étaient correctement lavés.

\section{Détermination des performances de reproduction}

Les escargots ont été élevés sur les différents substrats jusqu'à leur reproduction. Pendant les périodes de ponte, ces mollusques ont été régulièrement visités pour la vérification de la présence éventuelle d'œufs. La prospection des substrats a été pratiquée manuellement et quotidiennement. Les œufs trouvés simultanément à un même endroit (enfouis ou en surface de la litière d'élevage) ont été considérés comme constituant une ponte. Ces œufs ont été récoltés avec précaution à l'aide d'une cuillère en plastique et dénombrés par ponte. Leurs grands et petits diamètres ont été mesurés au millimètre près et leurs poids déterminés au $1 / 10$ de gramme près. Les œufs de chaque ponte ont été mis à incuber séparément à l'abri de la lumière dans un bac garni de bourres de noix de coco. Les substrats d'incubation contenant les œufs ont été légèrement arrosés une fois par semaine afin d'y maintenir une humidité relative constante. Après 24 jours d'incubation, les œufs sont régulièrement visités pour déceler les éclos et les sortir des bacs. Les éclos sont sortis des bacs car ils peuvent s'attaquer aux œufs non encore éclos empêchant ainsi leur évolution. Les éclos ont été dénombrés et les taux d'éclosion déterminés. Ainsi, les performances de reproduction des animaux sur chaque type de substrat ont été estimées à partir de l'âge de première ponte, du nombre de ponte par reproducteur, du nombre d'œufs par ponte, du poids, des grands et petits diamètres des œufs et du taux d'éclosion.

Nombre total de ponte /reproducteur $=$ Nombre total de ponte $/$ Nombre total de reproducteurs

Taux d'éclosion $=$ Nombre total d'éclos x $100 /$ Nombre total d'œufs pondus

\section{Résultats et Discussion Résultats}

Le nombre de ponte par reproducteur croit avec le taux d'amendement du substrat en poudre de coquille d'œufs de poule (Figure 6). Cette croissance du nombre de ponte par escargot devient significative à partir du taux d'amendement de $20 \%$. 


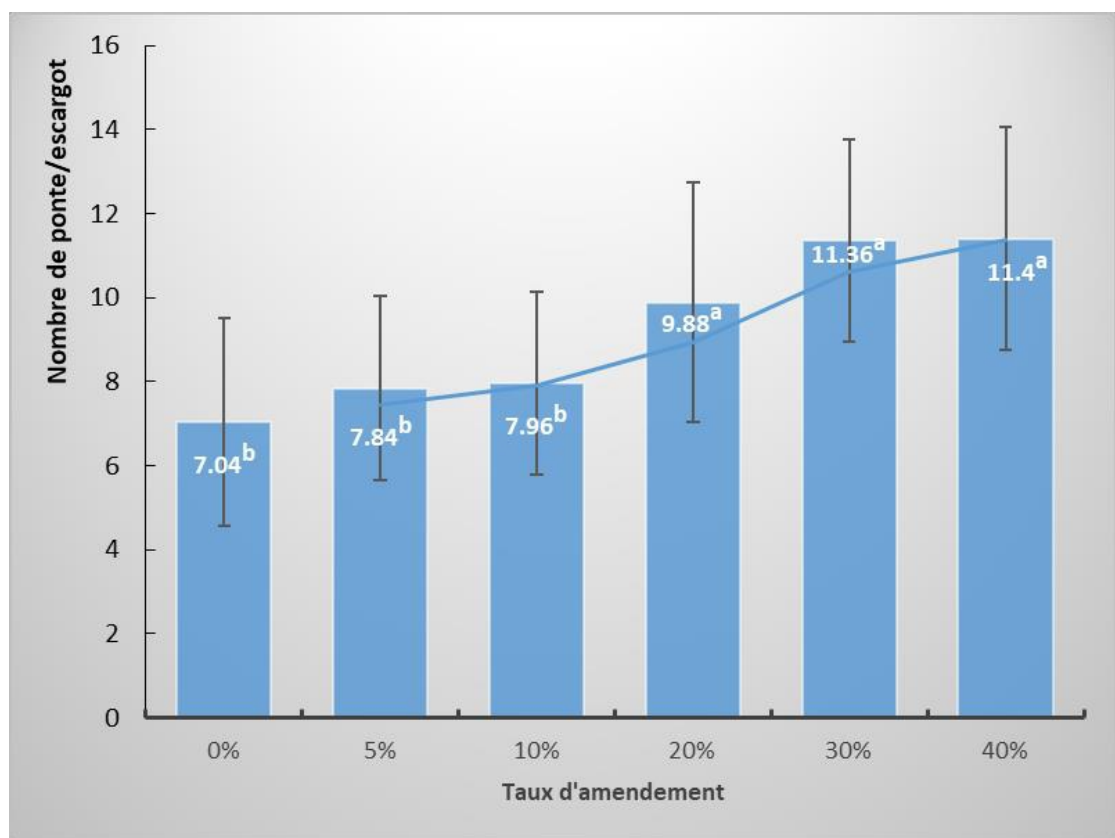

Figure 6: Nombre de ponte par reproducteur en fonction du taux d'amendement

Le nombre d'œufs par ponte croit aussi avec le taux d'amendement du substrat. Cette croissance du nombre d'œufs par ponte devient significative à partir du taux d'amendement de 30\%. Ainsi, les escargots élevés sur les substrats contenant $30 \%$ et $40 \%$ de poudre de coquille d'œufs ont donné plus d'œufs par ponte (figure 7).

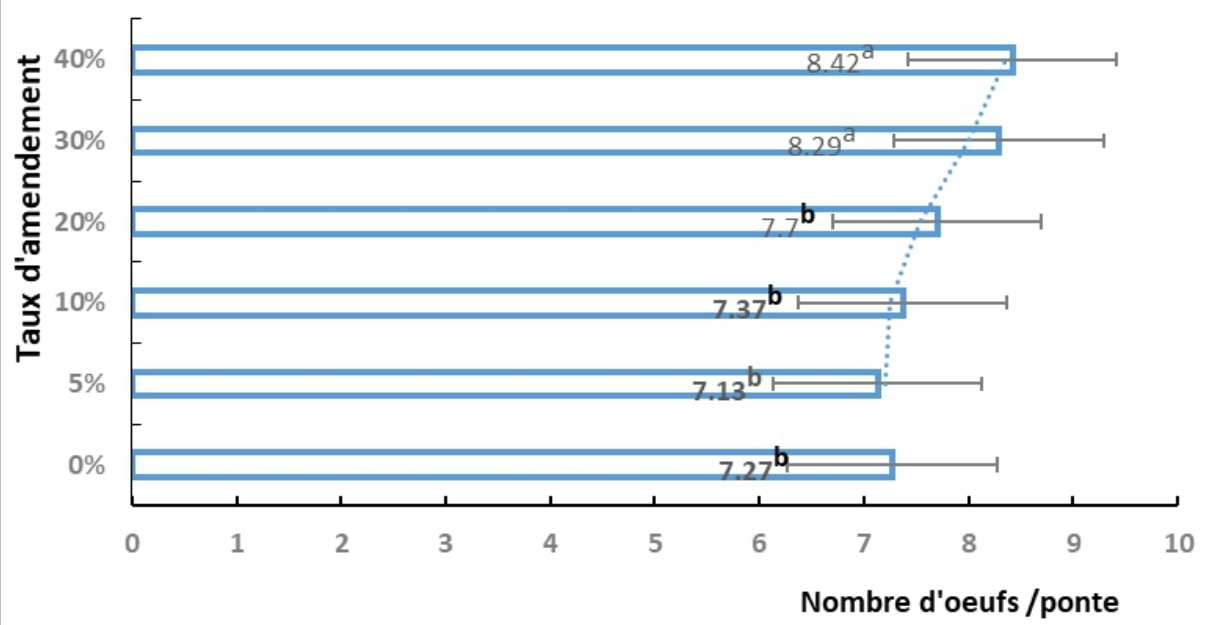

Figure 7: Nombre de ponte par reproducteur en fonction du taux d'amendement

Quant au poids moyen des œufs, il croit avec l'augmentation du taux d'amendement du substrat jusqu'à atteindre son pic au taux d'amendement de 
$20 \%$. Au-delà de $20 \%$ dans le substrat la source calcique entraine une baisse progressive du poids des œufs pondus (Figure 8).

\section{Poids moyen des oeufs (g)}

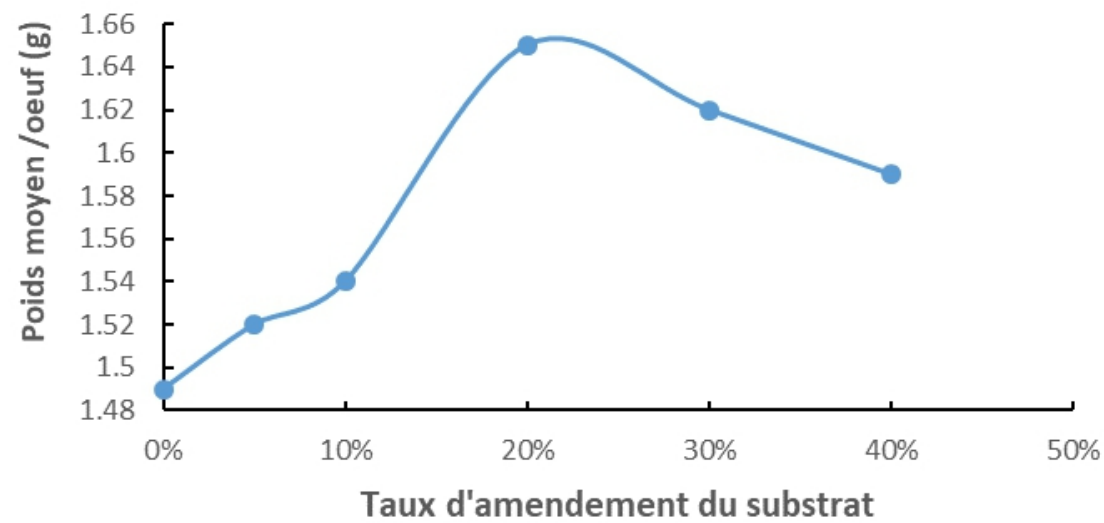

Figure 8 : Poids des œufs en fonction du taux d'amendement du substrat

D’une manière générale, les dimensions (grand et petit diamètres) des œufs s'augmentent avec le taux d'amendement du substrat d'élevage. Les œufs les plus gros sont pondus par les escargots des substrats amendés à un taux minimum de 10\% (Figure 9).

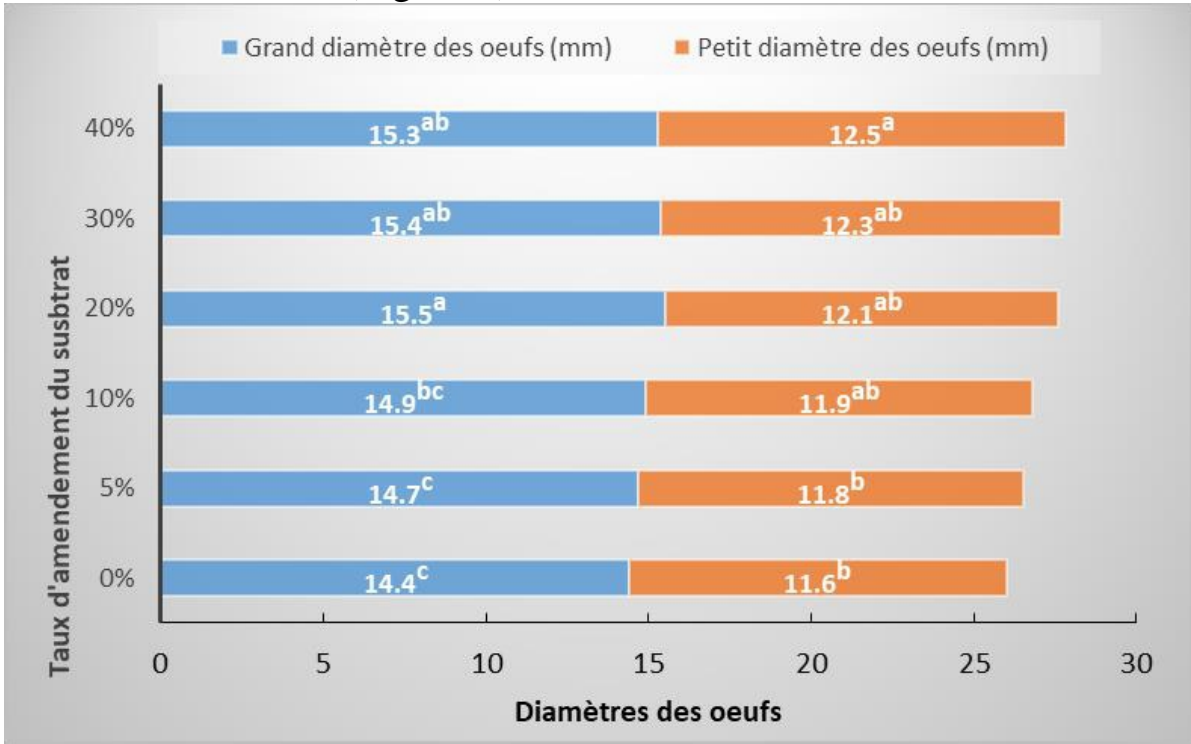

Figure 9 : Dimension des œufs en fonction du taux d'amendement du substrat

Les nombres d'éclos enregistrés sur les substrats amendés à $40 \%$ et 5\% représentent chacun $16 \%$ des éclos totaux dénombrés. Les quatre autres substrats ont enregistré chacun 17\% des éclos totaux (Figure 10). 


\section{Taux d'éclosion}

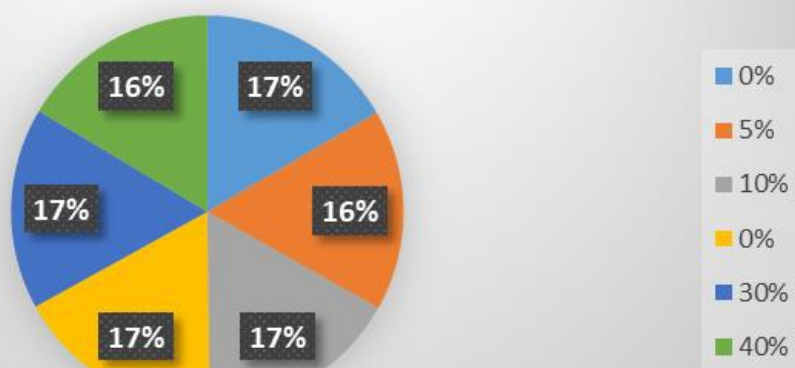

Figure 10 : Pourcentage d'éclos en fonction du taux d'amendement

\section{Discussion}

Il ressort de l'analyse des résultats que l'âge de première ponte des escargots diminue au fur et mesure que le taux d'amendement du substrat avec la poudre de coquilles d'œufs de poule croit. Ainsi, pendant que l'âge de première ponte est de 10,17 mois sur le substrat non amendé il est de 8,97 mois sur le substrat contenant $40 \%$ de la source calcique, soit une différence d'un délai de 1,2 mois. Les âges d'entrée en ponte enregistrés dans cette étude sont largement inférieurs à celui (36 mois) présenté par Dafem et al. (2008). Aussi, le nombre d'œufs par ponte $(7,27$ à 8,42$)$ et le poids moyen d'un œuf $(1,49$ à 1,65 ), enregistré sur tous les substrats au cours de cette étude sont supérieurs à ceux présentés par ces auteurs ; c'est-à-dire 6,39 pour le nombre d'œufs et $1,27 \mathrm{~g}$ pour le poids d'un œuf. Toutes ces différences seraient liées non seulement au fait que nos substrats d'élevage aient été amendés en source calcique contrairement à ces auteurs mais aussi au fait que nous ayons utilisé un aliment concentré riche en nutriment, notamment en calcium au lieu d'un aliment végétal. En effet, selon Otchoumou (2005) le taux de calcium alimentaire influence l'acquisition de la maturité sexuelle et la reproduction des escargots. Aussi, Otchoumou et al. (2011), affirment que l'escargot utilise une grande partie du calcium ingéré pour confectionner la coque de ses œufs. L'accroissement de la teneur en poudre de coquilles d'œufs de poule dans le substrat a induit une augmentation du nombre moyen annuel de ponte par reproducteur. En effet, le nombre de ponte annuel par escargot est passé de 7,27 sur le témoin à 8,42 sur le substrat contenant $40 \%$ de la source calcique. Aussi, l'amendement du substrat en source calcique a entrainé l'augmentation des caractéristiques physique (poids et dimensions) des œufs. Les légères variations du taux d'éclosion des oufs récoltés sur les différents substrats ne semblent pas être liées à leur teneur en source calcique. Cela serait plutôt lié 
au nombre d'œufs trouvés non enfouis, à la surface des litières lors des prospections et collectes pour incubation. En effet, Les œufs ainsi déposés à la surface des substrats lors de la ponte, sont exposés à l'air libre, ce qui entraîne leursèchement et leur craquement (Brisson, 1968).

\section{Conclusion}

La poudre de coquille d'œufs de poule convient pour l'amendement en calcium du substrat des reproducteurs d'escargots Archachatina marginata en captivité. L'amendement du substrat en cette source calcique permet non seulement de raccourcir le délai de maturité sexuelle des escargots mais aussi d'améliorer leurs performances de ponte. Le taux d'amendement convenable à cet effet est d'au moins $20 \%$.

\section{References:}

1. Aman J.B., KouassiK.D., Karamoko M.\& Otchoumou A. 2011. Effet de la teneur en poudre de coquille d'huitres dans le substrat d'élevage sur la croissance d'Archachatina marginata. Journal of applied Biosciences 47 : 3205-3213.

2. Bonnet J.C., Aupinel P. \& Vrillon J.L. 1990. L'escargot Helix aspersa : biologie-élevage. Institut National de la Recherche Agronomique, Editeur, Paris, ISBN 2 -7380-0247- 1, 124p.

3. Bouye t.r., Sika A., Memel J.D., Karamoko M. \& Otchoumou A., 2013. Effets de la teneur en poudre de coquilles de bivalves (Corbula trigona) du substrat sur les paramètres de croissance d'Achatina achatina (Linné, 1758) en élevage hors-sol. Afrique SCIENCES, 09 (2) : $142-153$

4. Brisson P., 1968. Développement de l'embryon et de ces annexes et étude en culture in vitro chez les achatines (Gastéropodes Pilmoés). Archives d'anatomie microscopique, 57(4) :345-368.

5. Chevalier H., 1985. L'élevage des escargots: production et préparation du petit gris. Edition du Points Vétérinaire, Paris 128p.

6. Conan L., Bonnet J. C. \& Aupinel P., 1989. L'escargot «petit-gris ». Progrès en alimention. Revue de l'alimentation animale, 3: 24-27.

7. Dafem R., Ngoula F., Teguia A., Kenfack A. \& Tchoumboué J., 2008. Performances de reproduction de l'escargot géant africain (Archachatina marginata) en captivité au Cameroun. Tropicultura, 26 (3) $155-158$.

8. Diomandé M., KIPRE A. V., Koussemon M. \& Kamenan A. 2008. Substitution de la farine de poisson par celle de l'escargots Achatina fulica dans l'alimentation des poules pondeuses en Côte d'Ivoire. Livestock Research for Rural 
Developpement,20(1)http/:www.cipav.org.co/Irrd/Irrd20/1diom2000 2.htm.

9. Hotopp K.P., 2002. Land snail and soil calcium in central Appalachian mountain forest.Southeasters Naturalist, 1: 27- 44.

10. Johannessen L.E. \& Solhoy T., 2001. Effects of experimental increased calcium levels in the litter on terrestrial snail populations. Pedobiologia, 45 (3) : 234-242.

11. Kouassi K.D., Otchoumou A. \& Dosso H., 2007. Les escargots comestibles de Côte d'Ivoire:influence de substrats d'élevage sur les paramètres de croissance de Archachatina ventricosa (Gould, 1850) en élevage hors sol. Tropicultura, 25 (1):16-20.

12. N'Da K., Otchoumou A., Koffi K. JC., 2004. Alimentation à base de produits du papayer et maturation ovocytaire chez Achatina fulica (Bowdich, 1820) en Côte d'Ivoire. Tropicultura, 22 (4) : 168-172.

13. Otchoumou A., N' Da K., Dosso H., \& Kouadio D.K., 2005. L'escargot géant africain Achatina fulica (Bowdich, 1820), peste potentielle pour les végétaux des forêts anthropisées de Côte d'Ivoire. Haliotis 34: 71-81.

14. Otchoumou A, Dupont-Nivet M \& Dosso H., 2011. Effects of diet quality and dietary calcium on reproductive performance in Archachatina ventricosa (Gould 1850), Achatinidae, under indoor rearing conditions. Invertebrate Reproduction \& Development/ISSN 0792Ŕ $4259 \quad$ print/ISSN $2157 \mathrm{R} 0272$. http://www.tandfonline.com/loi/tinv20.

15.Rousselet M. 1982. L’élevage des escargots. Edition du point vétérinaire, Paris, $132 \mathrm{p}$.

16.Sika N. A., Karamoko M., Bouye T.R., Otchoumou A. \& Kouassi K.P., 2015. Effet de la teneur en protéines alimentaires sur la croissance de l'escargot Achatina fulica (Bowdich, 1720). International Journal of Innovation and Applied Studies, 13 (1): 85-93.

17.Tatterfield P., Warui C.M., Seddon M.B. \& Kiringe J.W., 2001. Land snail faunas of afromontane forests of Mont Kenya: ecology, diversity and distribution petterns. Journal of Biogeography, 28, 7: 843-861. 